\title{
Influences of screening magnetic resonance imaging parameters on high-intensity focused ultrasound outcome for adenomyosis
}

This article was published in the following Dove Press journal:

Reports in Medical Imaging

\section{Nguyen Minh Duc \\ Huynh Quang Huy}

Department of Radiology, Pham Ngoc Thach University of Medicine, Ho Chi Minh City, Vietnam
Correspondence: Nguyen Minh Duc Department of Radiology, Pham Ngoc Thach University of Medicine, 86/2 Thanh Thai, Ward I2, District 10, Ho Chi Minh City, Vietnam

Tel +8490288 6899

Fax +84 38650025

Email bsnguyenminhduc@pnt.edu.vn

\begin{abstract}
Uterine adenomyosis is one of the most common benign pelvic diseases that adversely affects the quality of a woman's life. High-intensity focused ultrasound is an effective noninvasive treatment option for symptomatic adenomyosis. Clinical studies showed that the prediction of immediate treatment outcome, defined as nonperfused volume ratio for adenomyosis, is one of the key parameters for determining the therapeutic efficacy. MRI parameters of adenomyosis in the screening phase, such as type, location, abdominal subcutaneous fat thickness, signal intensity of adenomyosis, size, number of foci inside the lesion, and perfusion, manifest the impact on nonperfused volume ratio. Therefore, in this article, we performed a literature review of the influential screening MRI parameters on the immediate high-intensity focused ultrasound outcome. Keywords: adenomyosis, high-intensity focused ultrasound, magnetic resonance imaging, nonperfused volume ratio, treatment outcome
\end{abstract}

\section{Introduction}

Adenomyosis is a common and benign uterine disease that affects women of reproductive age. This disease is characterized by endometrial tissue of the endometrium layer invading the smooth muscle tissue of the myometrium layer, which leads to not only smooth muscle tissue hyperplasia around the lesion but also enlargement of the junctional zone. Adenomyosis often produces pelvic pain, a heavy menstrual cycle, and infertility, which seriously affect a woman's quality of life. The incidence of adenomyosis ranges from $5 \%$ to $70 \%$ due to differences in ethnicity. ${ }^{1-6}$

MRI is an optimal modality in localizing and featuring the junctional-zone width. ${ }^{1-6}$ On T2-weighted (T2W) images, a junctional-zone width $\geq 12 \mathrm{~mm}$ is the main criterion for diagnosing adenomyosis (Figure 1). ${ }^{1-6}$ In addition, MRI also has an important role in the planning and selection of treatment strategies, as well as monitoring the response of adenomyosis to therapies. ${ }^{7-9}$

A range of treatment options for symptomatic adenomyosis is available depending on the patient's age, type of adenomyosis, and desire for future fertility: gonadotropinreleasing hormone agonists (GnRHa), uterine artery embolization, high-intensity focused ultrasound (HIFU), uterus-sparing surgical treatment, and myometrial reduction. ${ }^{10}$ HIFU is a noninvasive ablation technique based on the thermal effects of sonication energy on target tissue, which is heated up to the temperature threshold of protein degeneration and tissue death. ${ }^{7,8,11}$ Several HIFU studies showed that patients with larger nonperfused volume (NPV) ratio, defined as NPV measured in perfusion MRI acquired immediately after HIFU treatment divided by adenomyosis volume 


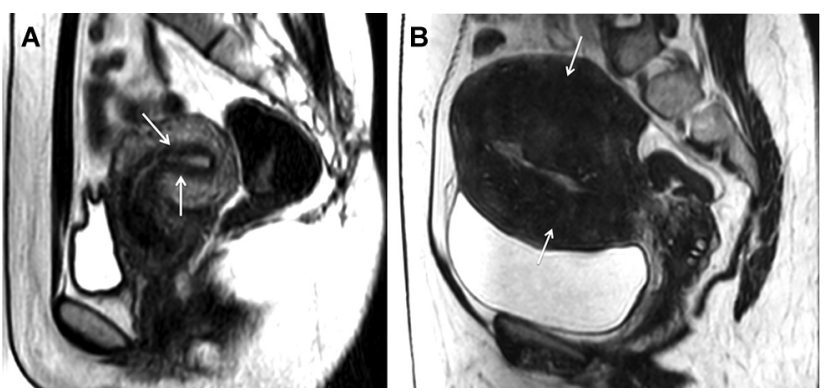

Figure I Sagittal T2-weighted MR images show (A) well-defined and normal junctional-zone width on both the anterior and posterior walls of a retroverted uterus (white arrows) and (B) ill-defined and widened junctional-zone width on both the anterior and posterior walls of an anteverted uterus (white arrows). Abbreviation: MR, magnetic resonance.

measured in $\mathrm{T} 2 \mathrm{~W}$ images acquired prior to treatment, had significantly greater symptom improvement, with higher probability of long-term sustained symptom relief. ${ }^{7,8,11}$ Because the MRI parameters of adenomyosis in the screening phase have an impact on the NPV ratio in treatment phase, ${ }^{7,8}$ we, therefore in this article, performed a literature review of the influential screening MRI parameters: the adenomyosis types as focal and diffuse, signal intensity (SI) of adenomyosis based on $\mathrm{T} 1$ perfusion and T2 images, location of adenomyosis, and the thickness of the subcutaneous fat layer of the anterior abdominal wall, size of adenomyosis and foci, on the immediate HIFU outcome defined as NPV ratio for adenomyosis.

\section{Methods}

The diagnostic pelvic MRI (Ingenia 1.5 Tesla, Philips Healthcare, the Netherlands) screening procedure was adopted to describe adenomyosis characteristics prior to HIFU treatment. T2W turbo spin echo parameters were repetition time (TR)/echo time (TE): $420 / 160 \mathrm{~ms}$; flip angle: $90^{\circ}$; resolution: $1.5 \times 1.5 \times 1.5 \mathrm{~mm}$; field of view (FOV): $250 \mathrm{~mm}^{2} ; 133$ sagittal slices; and sensitivity encoding (SENSE). Dynamic contrast enhancement MRI parameters were TR/TE: 10/4.5 ms; FOV: $240 \mathrm{~mm}^{2}$; flip angle: degree for post-contrast, 12 dynamics; and 20 axial slices.

The patient selection criteria for HIFU treatment for adenomyosis in previous studies can be summarized as follows: Inclusion criteria was premenopausal women aged 18-56 years who were diagnosed with symptomatic adenomyosis. Exclusion criteria were women with endometrial disease, pelvic endometriosis or uncontrolled systemic diseases; those with positive pregnancy test results; those with allergy to MRI contrast agents; those with surgical clips inside the treatment window, and those with suspected malignancy. ${ }^{7,8,11}$
In HIFU treatment, the beam of HIFU propagates through soft tissue creating localized high temperatures $\left(>55^{\circ} \mathrm{C}\right)$ for a few seconds within the target, thus producing irreversible cell damage and coagulative necrosis. Image guidance, under ultrasound or MRI, during HIFU treatment is critical for pretreatment planning, intraprocedural targeting, treatment monitoring, and immediate posttreatment assessment of therapeutic effect. ${ }^{7,8,11}$ During the ablation procedure, obstacles such as scars and bowel loops appearing inside the HIFU treatment window were resolved efficiently. ${ }^{7,8,11,12}$

\section{Screening MRI parameters Types and SI of adenomyosis}

Adenomyosis is classified as focal or diffuse (Figure 2). Focal adenomyosis is characterized as a localized low SI region inside the myometrium continuous to the junctional-zone width that causes asymmetrical enlargement of the uterus on T2W images. Diffuse adenomyosis is made up of numerous low T2 SI bands on a widened junctional zone that cause symmetrical enlargement of the uterus. ${ }^{1-6} \mathrm{Zhang}$ et al reported that the mean NPV ratio was $71.7 \% \pm 19.3 \%$ and $71.6 \% \pm 19.1 \%$ in the focal and diffuse adenomyosis groups, respectively ( $p$ $>0.05$ ). Although NPV ratio was not significantly different between 2 groups, the 3-month follow-up symptom improvement of focal adenomyosis group was significantly better than that of diffuse adenomyosis group. ${ }^{7}$

Low T2 SI inside an adenomyosis lesion is explained by decreasing vascularity, thus lacking the higher SI of normal smooth muscle tissue, and ectopic gland cysts inside the lesion. Meanwhile, high T2 SI inside an adenomyosis lesion is manifested as angiogenesis and hypermoisture content. Gong et al found that T2 SI of adenomyosis has a linear relationship with the energy efficiency factor, which is the amount of energy required for tissue ablation per unit volume. ${ }^{8}$

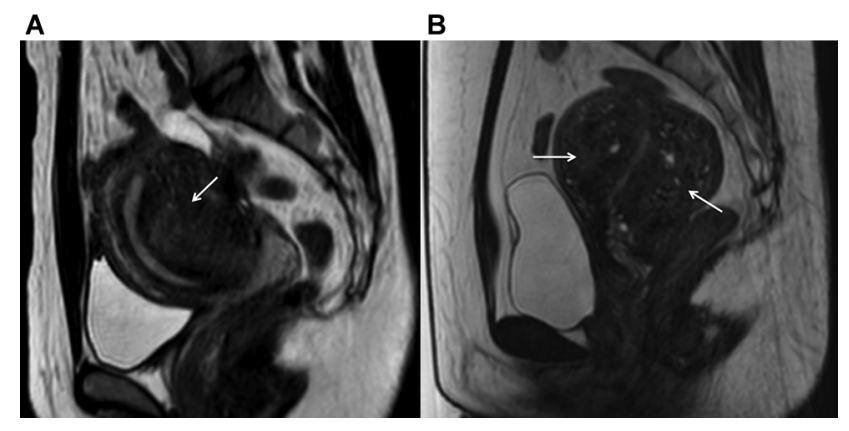

Figure 2 Sagittal T2-weighted MR images show (A) focal adenomyosis on the posterior wall of an anteverted uterus (white arrows) and (B) diffuse adenomyosis with some hyperintense foci on both the anterior and posterior walls of a retroverted uterus (white arrows).

Abbreviation: MR, magnetic resonance. 


\section{Location of adenomyosis and abdominal subcutaneous fat thickness}

With an increasing amount of tissues in front of the target location, ultrasonic energy is consequently attenuated because tissues in the sonication pathway absorb, reflect, and scatter ultrasonic waves. ${ }^{7,8,13,14}$

Adenomyosis can be located on the fundus or anterior wall, which is near the abdominal wall compared with the posterior wall of the uterus (Figure 3). The optimal distance in MRI-guided HIFU treatment from the skin to the lesion is $12 \mathrm{~cm}$. In problematic cases, where adenomyosis is located on the posterior wall of a retroverted uterus, the patient's anatomical manipulation should be utilized to gain maximal access to the target area prior to HIFU ablation. ${ }^{11,16}$

According to Gong et al study, the NPV ratio correlated with the location of adenomyosis on the uterus and the uterine position and was significantly higher in patients with adenomyosis on the anterior wall of an anteverted uterus compared with patients with adenomyosis on the posterior wall of a retroverted uterus. ${ }^{8}$ This could be explained by the sonication energy passing through a shorter distance for the anterior wall of an anteverted uterus than for the posterior wall of a retroverted uterus; therefore, the ultrasound energy is less debilitated along the pathway from the source to the target area. In addition, lesions on the anterior wall or fundus of the uterus can take advantage of the gradual heataccumulation phenomenon, which is the cumulative energy deposition of overlapped sonication beam during ablation procedure. ${ }^{7,8,13-15}$

Due to the absorption and distortion of sonication energy by adipose tissue, abdominal subcutaneous fat thickness is one of the significant parameters negatively correlated with the NPV ratio. ${ }^{14,15}$ Thus, patients with abdominal subcutaneous fat thickness $\geq 5 \mathrm{~cm}$ are not recommended for HIFU ablation, as there is a higher risk of subcutaneous fat burn and a lower chance of achieving high NPV ratio. ${ }^{17-19}$

\section{Foci}

The appearance of foci inside a lesion is commonly supported by the diagnosis of adenomyosis. ${ }^{1-6}$ Low SI foci on $\mathrm{T} 1 \mathrm{~W}$ images are seen for non-hemorrhagic ectopic endometrial gland cysts. Meanwhile, high SI foci on both T1W and T2W images are seen for hemorrhage or high-protein-concentration cysts (Figure 4). ${ }^{4-9}$ Different biological elements of high SI foci inside adenomyosis lesions produce a heterogeneous surface at the target point and lead to arduous sonication deposit as well as swift sonication dispersion during the ablation procedure. ${ }^{7,8,13-15}$

Gong et al reported that an adenomyosis lesion with multiple hyperintense foci is more difficult to ablate than adenomyosis with fewer hyperintense foci (Figure 5). The NPV ratio obtained in the group with fewer hyperintense foci was significantly higher than in the group with multiple hyperintense foci, with similar sonication power and treatment time. The results suggested that the quantity of hyperintense foci is considered as a predictor of the response of the adenomyosis to HIFU treatment. ${ }^{15}$

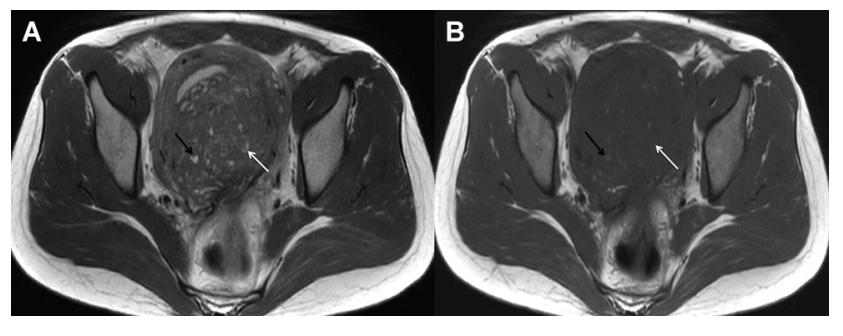

Figure 4 Sagittal T2-weighted MR image (A) shows focal adenomyosis on the posterior wall of a uterus with numerous hyperintense foci inside the lesion (white arrow and black arrow), and axial TI-weighted MR image (B) shows some hyperintense spots (black arrow) and some hypointense spots (white arrow) corresponding to all hyperintense foci on the T2-weighted image.

Abbreviation: MR, magnetic resonance.
A

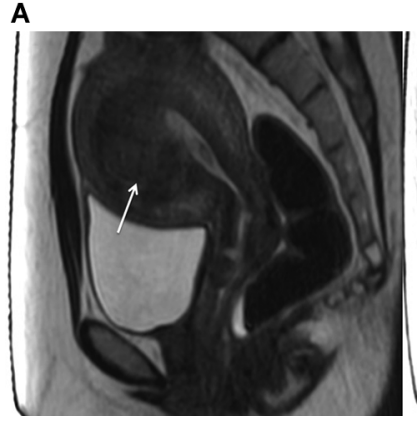

B
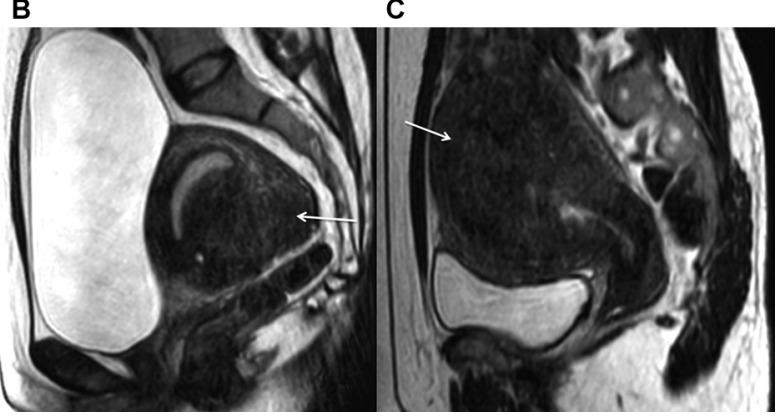

Figure 3 Sagittal T2-weighted MR images show (A) focal adenomyosis on the anterior wall of an anteverted uterus (white arrow); (B) focal adenomyosis on the posterior wall of a retroverted uterus (white arrow); and (C) diffuse adenomyosis on the fundus of an anteverted uterus (white arrow).

Abbreviation: MR, magnetic resonance. 


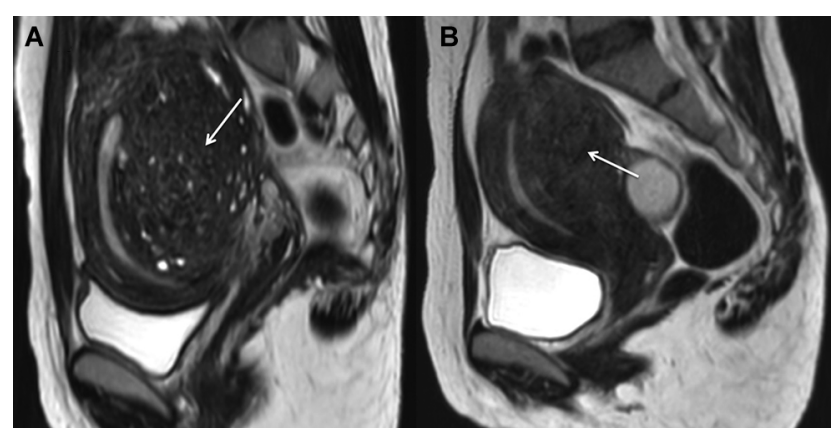

Figure 5 Sagittal T2-weighted MR images show (A) focal adenomyosis on the posterior wall of an anteverted uterus with numerous hyperintense foci inside the lesion (white arrow) and (B) focal adenomyosis on the posterior wall of an anteverted uterus without hyperintense foci inside the lesion (white arrow). Abbreviation: MR, magnetic resonance.

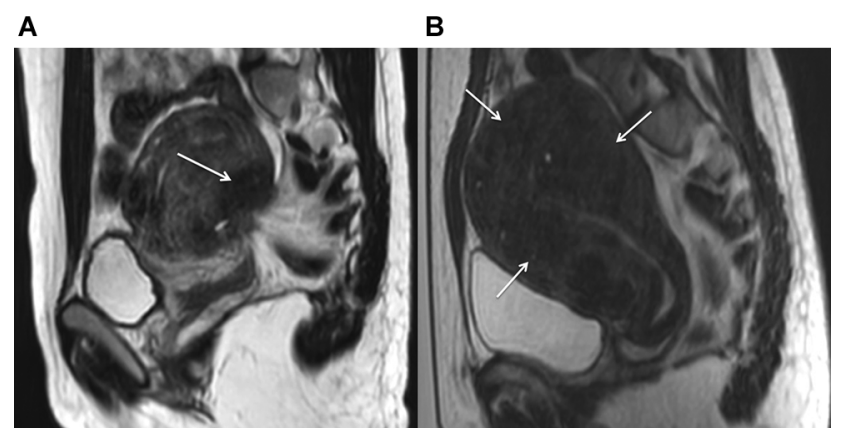

Figure 6 Sagittal T2-weighted MR images show (A) small focal adenomyosis on the posterior wall of a retroverted uterus (white arrow) and (B) large diffuse adenomyosis resulting in increased uterine volume (white arrows).

Abbreviation: MR, magnetic resonance.

\section{Size}

Adenomyosis size can vary from $1 \mathrm{~cm}$ to several centimeters (Figure 6). Some studies stated that adenomyosis diameters indicated for HIFU treatment can range commonly from 3 to $10 \mathrm{~cm} \cdot{ }^{17,19}$ Recently, Long et al showed that HIFU treatment was safe and effective for patients with a uterine adenomyosis volume $\geq 200 \mathrm{~cm}^{3}$ without severe complications. ${ }^{20}$ Some studies conclude that the volume of adenomyosis is positively correlated with the NPV ratio. ${ }^{8,14,15}$

In a study by Xiao-Ying et al, diffuse adenomyosis patients with the volume of the uterus $\geq 300 \mathrm{~cm}^{3}$ received 3 consecutive months of GnRHa prior to HIFU ablation were considered as HIFU plus GnRHa group. The results revealed that even adenomyosis volume in the HIFU plus GnRHa group was higher than the HIFU-only group, the NPV ratio, sonication power, and treatment time were significantly better than those in the HIFU-only group. Furthermore, the follow-up symptom relief rate was significantly higher in the HIFU plus GnRHa group than the HIFU group. ${ }^{21}$
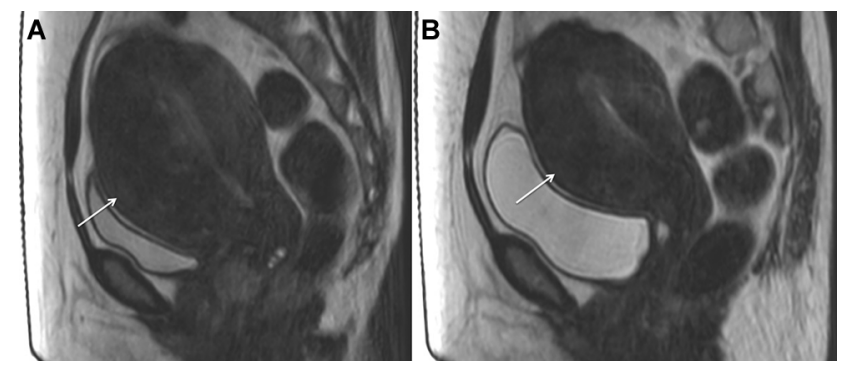

Figure 7 Sagittal T2-weighted MR images show (A) focal adenomyosis resulting in an increase of both the anterior wall thickness and the volume of the uterus (white arrow) and (B) after utilizing a 3-month GnRHa pre-HIFU treatment, combined with HIFU, both the volume of the uterus and the lesion thickness are reduced (white arrow).

Abbreviations: GnRHa, gonadotropin-releasing hormone agonists; HIFU, highintensity focused ultrasound; MR, magnetic resonance.

In a study by Guo et al, 55 patients in the group were treated with HIFU ablation only, versus 24 patients in the group who underwent HIFU ablation combined with preHIFU treatment of a 3-month GnRHa. The mean NPV ratio was not significantly different between the 2 groups. However, at the 3- and 6-month follow-up, symptoms of menstrual blood loss, uterine volume, and adenomyosis thickness were significantly more reduced in the group that utilized both HIFU ablation and the GnRHa pre-HIFU treatment (Figure 7). ${ }^{22}$ They concluded that GnRHa pre-HIFU treatment can not only reduce the size of both uterine volume and adenomyosis thickness but also the perfusion statement of lesions, thus ensuring the safety profile and efficacy of ablation. $^{22}$

In another study by Hou et al, patients with large adenomyosis volume who underwent HIFU ablation were divided into 2 groups: single HIFU group and HIFU plus metformin group. The findings demonstrated that follow-up inflammatory cytokine concentration (interleukin-1, 6, and 8), cancer antigen 125, menstrual volume, and pain of HIFU plus metformin group were significantly better than those of single HIFU group, suggesting that combined HIFU and metformin treatment is more efficient than single HIFU ablation. ${ }^{23}$

\section{TI-perfusion imaging}

According to Pennes' equation, the most influential factor affecting the accumulation of heat is vascularity inside the tissue; therefore, the blood supply level of adenomyosis affects HIFU ablation efficacy. ${ }^{24}$ In one previous study, the results showed that the degree of enhancement clearly represented the blood supply of the adenomyosis. Strong enhancement in the arterial phase reflected a rich blood supply to the adenomyosis. ${ }^{8}$ They concluded that hyperenhanced adenomyosis lesion that was difficult to be ablated produced a low NPV 


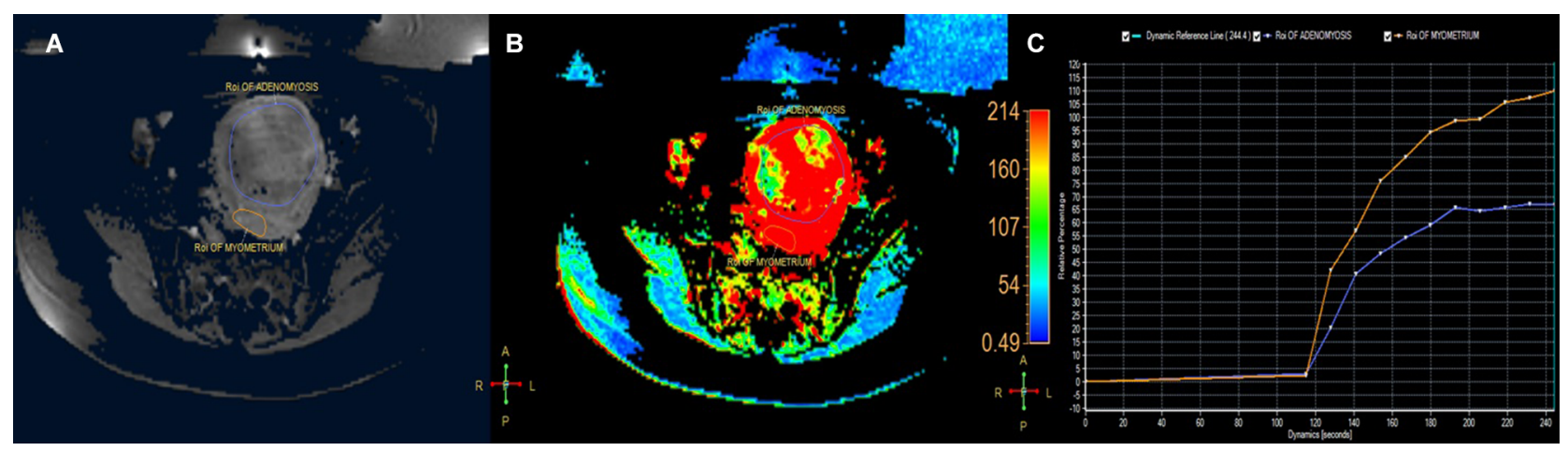

Figure 8 Region of interest within the area of the adenomyosis and the myometrium on one of the perfusion MR images (A); the perfusion map (B); and the time-SI curve of the adenomyosis is lower than that of the myometrium (C).

Abbreviations: MR, magnetic resonance; SI, signal intensity.

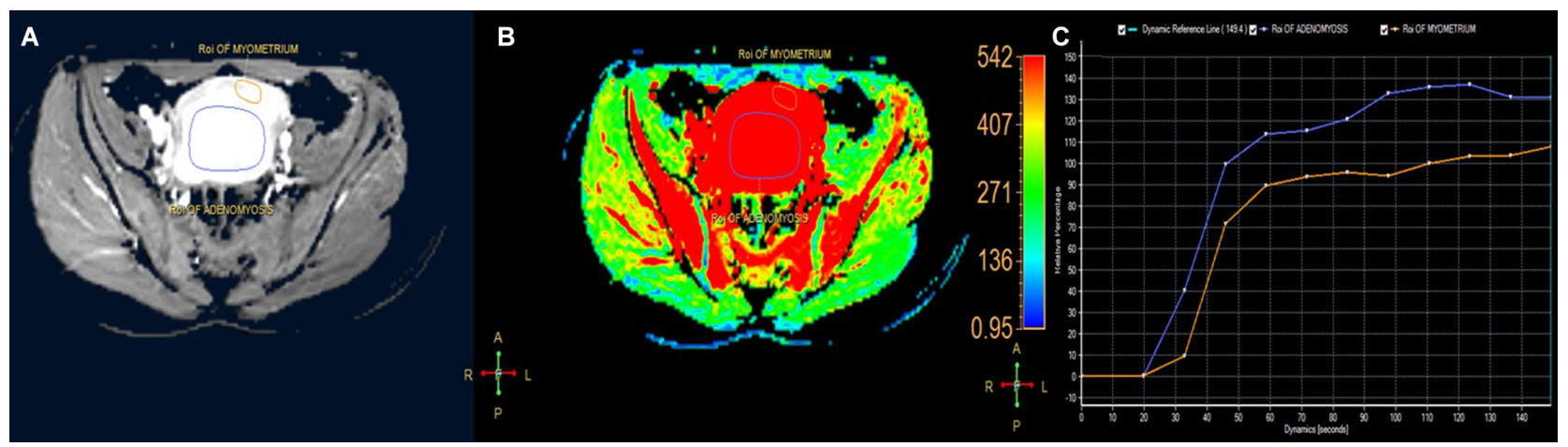

Figure 9 Region of interest within the area of the adenomyosis and the myometrium on one of the perfusion MR images (A); the perfusion map (B); and the time-SI curve of the adenomyosis is higher than that of the myometrium (C).

Abbreviations: MR, magnetic resonance; SI, signal intensity.

ratio due to strong arterial blood flow, which dissipates the thermal energy away from the focus point. ${ }^{8}$

In histopathology, the normal smooth muscle tissue of the myometrium is more perfused than adenomyosis, thus making it an effective internal reference for adenomyosis to be compared in terms of the perfusion condition. ${ }^{9,25}$ In a study by Keserci et al, adenomyosis was classified into 2 groups: group A with a time intensity curve of adenomyosis lower than the myometrium (considered as a weak perfusion group) (Figure 8) and group B with a time intensity curve equal to or higher than the myometrium (considered as a strong perfusion group) (Figure 9). The mean immediate NPV ratio and 6-month follow-up adenomyosis volume-reduction ratio in group A were significantly higher than those in group B. ${ }^{25}$ Therefore, understanding adenomyosis perfusion is one of the key references in HIFU ablation.

\section{Conclusion}

MRI can assist in treatment planning by determining the characteristics of adenomyosis prior to HIFU ablation. Based on the screening MRI parameters reviewed in this study, clinicians can clearly understand adenomyosis characteristics as well as effectively ablate adenomyosis for better HIFU outcome.

\section{Acknowledgment}

Written informed consent was obtained from all patients for the use of their images in this article.

\section{Disclosure}

The authors report no conflicts of interest in this article.

\section{References}

1. Mehasseb MK. Review Adenomyosis uteri: an update. Obstet Gynecol. 2009;11:41-47.

2. Struble J, Reid S, Bedaiwy MA. Adenomyosis a clinical review of a challenging gynecologic condition. J Minim Invasive Gynecol. 2016;23:164-185.

3. Novellas S, Chassang M, Delotte J, et al. MRI characteristics of the uterine junctional zone: from normal to the diagnosis of adenomyosis. AJR Am J Roentgenol. 2011;196:1206-1213.

4. Byun JY, Kim SE, Choi BG, Ko GY, Jung SE, Choi KH. Diffuse and focal adenomyosis. Radiographics. 1999;19:161-170.

5. Takeuchi M, Matsuzaki K. Adenomyosis: usual and unusual imaging manifestations, pitfalls, and problem-solving MR imaging techniques. Radiographics. 2011;31:99-115. 
6. Tamai K, Togashi K, Ito T, Morisawa N, Fujiwara T, Koyama T. MR Imaging findings of adenomyosis: correlation with histopathologic features and diagnostic pitfalls. Radiographics. 2005;25:21-40.

7. Zhang X, Li K, Xie B, He J, Zhang L. Effective ablation therapy of adenomyosis with ultrasound-guided high-intensity focused ultrasound. Int J Gynaecol Obstet. 2014;124:207-311.

8. Gong C, Yang B, Shi Y, et al. Factors influencing the ablative efficiency of high intensity focused ultrasound (HIFU) treatment for adenomyosis: a retrospective study. Int J Hyperthermia. 2016;32:496-503.

9. Mark AS, Hricak H, Heinrichs LW, et al. Adenomyosis and leiomyoma: differential diagnosis with MR imaging. Radiology. 1987;163: $527-529$.

10. Soave I, Wenger JM, Pluchino N, Marci R. Treatment options and reproductive outcome for adenomyosis-associated infertility. Curr Med Res Opin. 2017;10:1-11.

11. Rueff LE, Raman SS. Clinical and technical aspects of MR guided high intensity focused ultrasound for treatment of symptomatic uterine fibroids. Semin Intervent Radiol. 2013;30:347-353.

12. Keserci B, Duc NM. Volumetric magnetic resonance-guided high-intensity focused ultrasound ablation of uterine fibroids through abdominal scars: the impact of a scar patch on therapeutic efficacy and adverse effects. J Ther Ultrasound. 2017;5:22.

13. Fry FJ. Intense focused ultrasound in medicine. Some practical guiding physical principles from sound source to focal site in tissue. Eur Urol. 1993;23:2-7.

14. Zhang L, Rao F, Setzen R. High intensity focused ultrasound for the treatment of adenomyosis: selection criteria, efficacy, safety and fertility. Acta Obstet Gynecol Scand. 2017;96:707-714.

15. Gong C, Setzen R, Liu Z, et al. High intensity focused ultrasound treatment of adenomyosis: the relationship between the features of magnetic resonance imaging on T2 weighted images and the therapeutic efficacy. Eur J Radiol. 2017;89:117-122.
16. Froling V, Kroncke TJ, Schreiter NF, et al. Technical eligibility for treatment of magnetic resonance-guided focused ultrasound surgery. Cardiovasc Intervent Radiol. 2014;37:445-450.

17. Lee JS, Hong GY, Park BJ, Kim TE. Ultrasound-guided high-intensity focused ultrasound treatment for uterine fibroid and adenomyosis: a single center experience from the Republic of Korea. Ultrason Sonochem. 2015;27:682-687.

18. Fan TY, Zhang L, Chen W, et al. Feasibility of MRI-guided high intensity focused ultrasound treatment for adenomyosis. Eur J Radiol. 2012;81:3624-3630.

19. Cheung VYT. Current status of high-intensity focused ultrasound for the management of uterine adenomyosis. Ultrasonography. 2017;36: 95-102.

20. Long L, Chen J, Xiong Y, et al. Efficacy of high-intensity focused ultrasound ablation for adenomyosis therapy and sexual life quality. Int J Clin Exp Med. 2015;8:11701-11707.

21. Xiao-Ying Z, Ying-Shu G, Jiu-Mei C, et al. Effect of pre-treatment with gonadotropin-releasing hormone analogue GnRH- on high-intensityfocussed ultrasound ablation for diffuse adenomyosis: a preliminary study. Int J Hyperthermia. 2018;2:1-9.

22. Guo Y, Duan H, Cheng J, Zhang Y. Gonadotrophin-releasing hormone agonist combined with high-intensity focused ultrasound ablation for adenomyosis: a clinical study. BJOG. 2017;124:7-11.

23. Hou Y, Qin Z, Fan K, Xu Y, Huang X. Combination therapeutic effects of high intensity focused ultrasound and metformin for the treatment of adenomyosis. Exp Ther Med. 2018;15:2104-2108.

24. Pennes HH. Analysis of tissue and arterial blood temperatures in the resting human forearm. J Appl Physiol. 1948;1:93-122.

25. Keserci B, Duc NM. Volumetric magnetic resonance guided focused ultrasound ablation: the role of dynamic contrast enhanced magnetic resonance imaging in classifying adenomyosis and predicting the immediate treatment outcome. Int J Hyperthermia. 2017;19:1-9.
Reports in Medical Imaging

\section{Publish your work in this journal}

Reports in Medical Imaging is an international, peer-reviewed, open access journal publishing original research, reports, reviews and commentaries on all areas of medical imaging. The manuscript management system is completely online and includes a very quick and fair peer-review system, which is all easy to use.

\section{Dovepress}

Visit http://www.dovepress.com/testimonials.php to read real quotes from published authors. 